\title{
Determination of Brain Death: An Overview with a Special Emphasis on New Ultrasound Techniques for Confirmatory Testing
}

\author{
Albrecht Günther ${ }^{*}, 1$, Hubertus Axer ${ }^{1}$, Juan-Antonio Llompart Pou ${ }^{2}$, Otto W. Witte ${ }^{1}$ and \\ Christoph Terborg ${ }^{3}$ \\ ${ }^{I}$ Department of Neurology, Friedrich-Schiller-University Jena, Germany \\ ${ }^{2}$ Intensive Care Unit, Hospital Universitari Son Dureta Palma de Mallorca, Illes Balears, Spain \\ ${ }^{3}$ Department of Neurology, Asklepios Klinik St Georg, Hamburg, Germany
}

\begin{abstract}
Progress in resuscitation medicine and the widespread success of cardiopulmonary support in intensive care medicine called for new standards for determining a patient's death. Following a worldwide discussion on the medical, ethical, and legal aspects of death, the term brain death is now a generally accepted criterion for death. Brain death, defined as complete and irreversible cessation of brain function was established to describe an individuals death even during artificial ventilation and a temporary maintenance of cardiac function in the ICU setting. The determination of brain death requires adequate prerequisites, as well as clinical findings verifying a complete loss of function of the entire brain, and a confirmation of its irreversibility.

Most countries have national guidelines aimed at providing a high-quality medical standard and to exclude any conflicts of interest. On confirmation of brain death, life-sustaining therapies are discontinued in accordance with the declared or assumed will of the dead individual. Only in cases of an intended organ donation, organ protective measures need to be continued until surgery.

In this overview, we aim to outline the components of determining clinical brain death with a special emphasis on new ultrasound techniques for confirmatory testing.
\end{abstract}

Keywords: Brain death, confirmatory testing, ultrasound, cerebral circulatory arrest.

\section{INTRODUCTION}

The diagnosis of clinical death was traditionally made by confirming a deep coma, apnea, pulselessness, muscle relaxation, pupillary dilatation and an absence of cerebral reflexes. However, these phenomena may be reversible in cases of a "vita minima" or "brain death mimics" [1]. The clinical signs of definite death comprise livor mortis, rigor mortis, and autolysis.

The progress in modern medicine over the last decades required new standards determining when human life ends and death begins. Even in patients with complete and irreversible brain damage, intensive care therapy with artificial ventilation, the use of catecholamines and other drugs, for example, insulin or vasopressors can preserve cardiocirculatory, pulmonary and endocrinological function for several days. Such life-prolonging and preserving measures raised the question as to the point of time when a patient is dead and thus, therapy is discontinued.

A definition of irreversible coma was initially reported by the Ad Hoc Committee of the Harvard Medical School in 1968 as a criterion defining brain death [2,3]. In the

\footnotetext{
*Address correspondence to this author at the Department of Neurology, Friedrich-Schiller-University Jena, Erlanger Allee 101, D-07747 Jena, Germany; Tel: ++49 +3641 9323417; Fax: ++49 +3641 9323402; E-mail: albrecht.guenther@med.uni-jena.de
}

intervening time following a broad discussion on the medical, ethical, and legal aspects, the concept of brain death signifying an individual's death has been further developed and accepted worldwide. This is reflected by national brain death practice guidelines in 70 of 80 countries systemically investigated by Wijdicks [4]. The concept denotes that the complete and irreversible loss of function of the entire brain constitutes a definite sign of death. This has important consequences for discontinuation of intensive care therapy or the institution of organ protective management with an aim towards successful organ donation.

\section{PATHOPHYSIOLOGY OF BRAIN DEATH}

Severe head trauma, intracranial haemorrhage (e.g. subarachnoid, subdural, epidural, intraparenchymateous, intraventricular), space-occupying ischemic stroke and hypoxic brain damage are frequent etiologies of brain death [5].

In accordance with the formal pathogenesis and the location of the primary injury, a complete and irreversible brain injury can be caused by primarily supratentorial or infratentorial damage, and global damage (secondary brain injury). However, this distinction is not mandatory worldwide [4]. Even though the location of the primary injury may be diverse, an increase in intracranial pressure (ICP) with subsequent circulatory arrest is common in brain death. The temporal dynamics of intracranial pressure depends on its etiology and severity. 
In principle, an increased intracranial volume owing to a local or global swelling does not directly increase ICP. However, the mechanisms to compensate for an increased intracranial volume are limited. According to the MonroKellie doctrine, when the fontanelles are closed and the skull is intact, an increase in intracranial volume, due, for example to intracerebral hemorrhage can only be compensated by alterations in cerebral blood volume or liquor volume. If a critical intracranial volume is reached, ICP exponentially increases with a decrease in cerebral blood flow, subsequent vasodilation when cerebral autoregulation is preserved, and an increase in cerebral blood volume. This is a vicious circle that leads to further impairment of cerebral perfusion. When the ICP exceeds the mean arterial blood pressure, cerebral perfusion pressure decreases towards zero concluding in a cerebral circulatory arrest that characterizes brain death [6].

In cases of supratentorial brain injuries, e.g. head trauma or space-occupying hemispheric stroke, the primary lesion is characterized by local bleeding, ischemia, perifocal edema, inflammatory processes, and subsequent hypoperfusion of the surrounding cerebral tissue. This may result in a progressive edema with a space-occupying effect, midlineshift, compression of basal cerebral arteries, and a transtentorial herniation followed by hydrocephalus, and a secondary ischemia of the infratentorial parts of the brain. As soon as the ICP rises above the mean arterial blood pressure, circulatory arrest results in global cerebral hypoxic-ischemic injury.

If the primary brain injury is located infratentorially, such as in pontine haemorrhage or basilar artery occlusion with vertebrobasilar infarction, the infratentorial ICP increases in conjunction with a decreased infratentorial blood flow with subsequent lesion growth through edema. This may result in upward transtentorial herniation and occlusive hydrocephalus, a subsequent rise in supratentorial ICP, and finally cerebral circulatory arrest.

Thirdly, global cerebral edema due to hypoxic-ischemic encephalopathy usually results from cardiac arrest and prolonged cardiac resuscitation or asphyxia. In this case, all parts of the brain experience hypoxic-ischemic damage. Typically, global cerebral ischemia results in cytotoxic edema and inflammation, which increases ICP and decreases cerebral blood flow (CBF) within a few days. If the elevated ICP exceeds the mean arterial blood pressure, cerebral perfusion comes to a halt with a complete and irreversible loss of brain function.

\section{DETERMINATION OF BRAIN DEATH}

Determination of brain death consists of three steps: 1) evaluation of the etiology of a severe brain injury and an exclusion of reversible causes, and 2) the clinical findings of deep coma, the lack of brain stem reflexes, and absence of a breathing drive (i.e. apnea), and 3 ) the confirmation of the irreversibility of these findings, either by repetitive examinations after a time interval, or by means of ancillary tests.

In many countries special recommendations for the determination of brain death have been published and the term brain death is generally accepted $[7,8]$. However, there is no consensus regarding the diagnostic criteria, and methods vary between different countries and institutions, so that physicians should be aware of the differences in legal procedures and local policies [9]. Thus, a standardised diagnostic procedure was urgently necessary and guidelines determining brain death in adults were for example, proposed by the American Academy of Neurology in 1995 [7].

In clinical practice, a careful neurological examination to determine brain death is necessary, which can best be undertaken by neurologists and neurosurgeons, although any physician with special expertise in neuro-intensive care and knowledge of brain death criteria should also be able to perform this clinical evaluation. However, physicians have to be aware of special diagnostic pitfalls, such the locked-in syndrome, Guillain-Barré-syndrome, intoxication, analgosedation, and other brain death mimics. A brain death confirmation performed by a second physician is required in many countries to exclude a potential diagnostic error. Finally, in order to avoid any conflicts of interest, the determination of brain death should not be performed by a transplantation surgeon, or a member of the transplantation team.

\section{Prerequisites}

The question of brain death after a severe brain injury frequently arises, if coma persists in a ventilated patient in the ICU setting without or after withdrawal of analgosedative agents. In a recently published series, the diagnosis of brain death was declared in $30 \%$ of patients within the first 24 hours and in $62 \%$ within the first 3 days after admission [10].

The first diagnostic step consists of clarifying the etiology of the coma and determining whether the severity of the brain injury is sufficient so as to result in a complete and irreversible loss of brain function. The diagnosis has to be carefully made by means of interpreting the medical history and the cerebral imaging, usually cranial computer or magnetic resonance tomography. The most frequent findings of catastrophic brain injuries include severe head trauma, subarachnoid hemorrhage, intracerebral hemorrhage, spaceoccupying ischemic stroke, or severe brain edema from other causes. In cases of hypoxic-ischemic encephalopathy, the neuroimaging findings may not be impressive, thus calling for special criteria to confirm the irreversibility of the complete loss of brain function. A routine examination of cerebrospinal fluid is not mandatory, however, it should be performed if an infection of the CNS is thought to be responsible for the clinical syndrome.

The next step must exclude potentially reversible causes of deep coma and loss of brain stem function. Acute arterial hypotension has to be ruled out, and the systolic arterial blood pressure should be $\geq 90 \mathrm{mmHg}$, eventually with the help of fluid resuscitation or vasopressors. Hypothermia may mimic complete and irreversible brain injury, therefore the body temperature should be above $32^{\circ} \mathrm{C}$ or $90^{\circ} \mathrm{F}$. Drug intoxication, if suspected, should be excluded by toxicological tests (barbiturates, tricyclic antidepressants, opiates, benzodiazepines, neuromuscular blocking agents or illegal drugs), and the effect of specific antidotes. If the drug or poison is known, an observational period of at least fourtimes the elimination half-time of the substance, or, if the substance is unknown, a period of 48 hours is recommended 
[1]. However, in case of doubt, either repetitive examinations after a certain time interval are necessary, or the cessation of cerebral circulation has to be proved. An acute and severe metabolic cause of coma (glucose, electrolytes, acid-base, or endocrine disturbances) has to be excluded by laboratory tests even though cut-off values which would definitely influence clinical brain death criteria are not known (see below). In these cases, confirmatory tests are required.

\section{Clinical Evaluation of Brain Death $[1,11]$}

\section{Presence of Deep Coma}

Deep coma means an absence of eye opening or motor reaction on verbal and painful stimuli. Painful stimuli include applying pressure on the sternum, the nail beds or on the supraorbital nerve.

\section{Absence of Brainstem Reflexes}

In brain death, both pupils are mid-dilated or dilated (4-9 $\mathrm{mm}$ ) and do not react to bright light. There is no eye movement during rapid head movements (oculocephalic reflex; test only when no fracture or cervical spine instability is present), or ice irrigation of the ear canal (oculovestibular reflex). In addition, the corneal reflex brought about by a cautious touching of the outer cornea is absent. Moreover, grimacing cannot be elicited by painful facial stimuli (pitfall: severe facial trauma), and a pharyngeal or tracheal reflex cannot be evoked by movements of the endotracheal tube or deep suctioning using a suction catheter.

\section{Presence of Apnea (Apnea Test)}

Apnea is the last brain stem malfunction that should be investigated, if the prerequisites are fulfilled and both deep coma and absence of the above mentioned brain stem reflexes are verified. The apnea test measures the reflectory respiration after an increase of $\mathrm{EtCO}_{2}$ due to hypoventilation or apnea. It requires a maintenance of $\mathrm{pO}_{2}$ by hyperoxygenation to avoid hypoxia. The procedure is safe, if an oxygen-diffusion technique with a hyperoxygenation phase and a disconnection phase is applied. During the hyperoxygenation phase, the $\mathrm{FiO}_{2}$ is elevated to 1.0 until the arterial $\mathrm{pO}_{2}$ rises above $200 \mathrm{mmHg}$. Through the disconnection or apnea phase, the patient is disconnected from the ventilator, but an oxygen catheter inserted deep in the trachea with $6-101 \mathrm{O}_{2} /$ min maintains arterial $\mathrm{pO}_{2}$ by diffusion even during apnea [12]. During the following 5 10 minutes, the patient's uncovered chest and abdomen are carefully observed for respiratory movements. It is useful to have good illumination in order to register each reaction, and to distinguish between visible movements of the thorax from the beating heart and respiratory movements. Alternatively, instead of the disconnection phase, the patient may remain connected to the ventilator with a low respiration rate of $2 /$ min and $\mathrm{FiO}_{2}$ of 1.0. Serial arterial blood gas analysis should be performed to monitor hypercarbia, however, in many countries a $\mathrm{pCO}_{2}$ target is not recommended [4]. If an arterial level above $60 \mathrm{mmHg} \mathrm{pCO}_{2}$ is reached without any respiratory movements, apnea is confirmed. If respiratory movements (clinically or on the respirator display) are visible, the complete loss of brain function, that is of the brain stem is not verified. If the apnea test is negative, it has to be aborted and the patient must immediately be reconnected to the ventilator.

There are a number of clinical observations compatible with a diagnosis of brain death: spontaneous limb movements, respiratory like movements (shoulder elevation and adduction, back arching), sweating, tachycardia, blood pressure fluctuations, Babinski and other reflexes of spinal origin [5]. Although, clinicians must be aware of spontaneous and reflex movements frequently occuring during the first 24 hours after brain death that do not exclude brain death. The most frequent movements include undulating toe flexion sign, triple flexion reflex (flexion of the thigh, leg, and foot), myoclonus as well as the so-called Lazarus sign, characterized by bilateral arm flexion, adduction of the shoulders, lifting of the arms and crossing of the hands (see also Table 1). However, if any clinical test regarding the presence of deep coma, absence of brain stem reflexes, or apnea is equivocal and brain death cannot be confirmed, repeat examinations are required.

\section{Confirmation of Irreversibility}

The confirmation of the irreversibility of the complete loss of cerebral function depends on the patient's age and the etiology of brain injury. Irreversibility can be confirmed by repeat investigations, ancillary tests, or both within an adequate observation period. Ancillary tests are not always mandatory [7] although, they allow the diagnosis of brain death even in hospitals without expertise in neurophysiological or brain perfusion procedures. However, it should be noted that there are substantial differences between the different national guidelines [4].

The following procedures in line with the German guidelines for determination of brain death take into aspect the patient's age and etiology of severe brain injury $[14,15]$ :

1a. For primary supratentorial brain injuries in adults, the diagnosis of brain death can be confirmed by repetitive determination of the prerequisites and the clinical syndrome (deep coma, brain stem areflexia and apnea) following a minimum observation period of 12 hours, or the performance of additional ancillary tests.

1b. In newborns and children until the age of 2 years, additional ancillary tests and a prolonged time of repetitive investigations are mandatory.

2. In primarily infratentorial brain injuries, which may cause a complete and irreversible loss of brain stem functions, the supratentorial parts of the brain have to be investigated by means of EEG or cerebral perfusion test to confirm brain death (see below).

3. In secondary brain injuries, usually in case of hypoxic-ischemic encephalopathy, it is necessary to determine the loss of complete brain function after a prolonged observation time of 72 hours with repeated clinical confirmations or by confirmatory testing (e.g. EEG, Doppler sonography).

\section{CONFIRMATORY TESTS}

Apart from repeat clinical evaluations, confirmatory tests to corroborate the cessation of brain activity are advocated in several countries particularly for patients in whom certain 
components of the prerequisites and clinical testing (e.g. apnea) or confounding factors (e.g. drug effects) cannot reliably be ascertained. In certain situation confirmatory tests are also employed to shorten brain death diagnostic procedures, and thus, the waiting time until possible organ transplantation, a procedure endorsed by many national guidelines.

In an ideal world, testing should give no false positive results, not be influenced by drugs or metabolic disturbances, be readily available on weekends and at night, and be safely applicable (without deleterious effects of the examination itself) in a standardized manner with a high level of accuracy across examiners and readers [16]. However, in the real world, none of the available confirmatory tests offer ideal characteristics to date. For example, EEG is strongly influenced by sedatives and other drugs, somatosensory evoked potentials (SSEP) can only be used if the peripheral nerves and the spinal tract are intact. In addition, testing for brainstem auditory evoked potentials (BAEP) requires an intact peripheral receptor and, moreover, ultrasound techniques are highly operator-dependent.

Confirmatory tests can, in general, be divided in cerebral perfusion studies and functional (electrophysiological) studies. Several tests are summarized in the section following with special emphasis placed on imaging aspects.

\section{Neurosonological Methods for Confirmatory Testing of Brain Death}

One of the most widely used methods for the determination of cerebral circulatory arrest (CCA) is the Doppler and/or duplex ultrasound technique. This safe, noninvasive, rapid and inexpensive procedure can be performed at the bedside [17]. Doppler sonographic evidence of CCA transpires when an oscillating flow (almost equal forward and reverse flows) or systolic spikes are detectable in every extracranial and basal cerebral artery (Fig. 2c). However, to date, there is no international consensus for the use of Doppler sonography including the need for an extracranial and a transcranial approach in the diagnosis of CCA as a confirmatory test. The finding of absent flow signals in the basal cerebral arteries in patients in whom a flow was previously detected before by the same investigator, and with extracranial findings corresponding to $\mathrm{CCA}$ can also confirm brain death. Then again, a missing signal from basal cerebral arteries in a single study could also result from an insufficient ultrasonic bone window. Thus, although transcranial Doppler (TCD) is highly specific, the method lacks in sensitivity delivering false negative results in up to $10 \%$ of cases [18]. Sonographic proof of a typical pattern of CCA may not be possible in cases with a poor acoustic window or where devastating intracranial injuries are present $[19,7]$. For such conditions, methods that enhance TCD sensitivity are a matter of current investigation. For example, some authors have reported on the use of transcervical or transorbital insonation and serial examinations with improved sensitivity [19-21], (see Fig. 1A, B). Cerebral blood flow signals might be absent if the blood pressure is low. The use of catecholamines to increase blood pressure might be a useful method to enhance cerebral blood flow signals when these are absent due to low blood pressure, a suggestion, not officially included in most brain death protocols.

The number of conclusive investigations, employing ultrasound contrast agent boluses to study cerebral perfusion in patients with suspected CCA have increased from $90 \%$ to 98\% according to Llompart-Pou et al. [22]. This functional sonographic, minimally invasive approach has recently been described in CCA assessment [23]. Furthermore, various ultrasound perfusion imaging techniques have also been developed [23-26], with phase harmonic perfusion imaging as representing the most relevant procedure (for review see [24]). Using a bolus-kinetic approach and time-intensity curves, different parameters of cerebral perfusion can be obtained: Of these, time-to-peak intensity appears to be the most accurate measure to evaluate cerebral perfusion, because of its low intra-individual variability and the lack of influence of the depth of the insonation plane. In ischemic stroke, a correlation between a delayed time-to-peak intensity (close to 4 seconds) and cerebral hypoperfusion in various regions of interest (ROI's) has been shown [27,28]. Accordingly, Abadal et al. [23] have recently developed an approach to diagnose CCA. In their pilot study including 13 patients with different neurocritical disorders leading to brain death, a bolus kinetic approach with a bolus injection of Sonovue ${ }^{\circledR}$ (Bracco SpA, Milan, Italy) was applied according to the protocol described by Krogias et al. [29]. Cerebral perfusion was studied in different predefined ROI's after a $2.5 \mathrm{ml}$ bolus injection of ultrasound contrast agent via a central line. In patients with demonstrated CCA and clinical diagnosis of brain death, a missing time-intensity curve in every ROI's was found (Fig. 1D). In contrast, in patients with minimal neurological response or even with high-resistance flow patterns a time-intensity curve was preserved with a delayed time-to-peak intensity (Fig. 1C). However, although promising, this study presented two limitations: firstly, it has not been systematically compared with other diagnostic modalities, and secondly, a single plane of insonation was employed, which does not depict the whole brain tissue.

In conclusion, according to preliminary studies, ultrasonographic cerebral perfusion imaging might allow a minimally invasive study of cerebral perfusion and may enhance the rate of CCA diagnosis with the proof of a characteristic cessation of perfusion.

Apart from cerebral blood flow imaging, the use of transcranial colour coded duplex sonography can also demonstrate oscillatory flow characteristics through changing of the colour spectra in the duplex image in conjunction with the Doppler signal showing oscillatory flow (Fig. 2). Furthermore, duplex sonography will in many cases, allow the distinction to be made between a missing acoustic bone window (no anatomical landmarks visible: $3^{\text {rd }}$ ventricle, mesencephalon, contralateral skull hyperdensity) and "no-flow" CCA in a brain dead patient.

\section{Other Cerebral Perfusion Techniques for Brain Death Confirmation}

Other emerging techniques include four-vessel intracranial digital subtraction angiography which utilises a radiocontrast agent as the traditional reference standard to confirm brain death by ascertaining CCA. The typical 

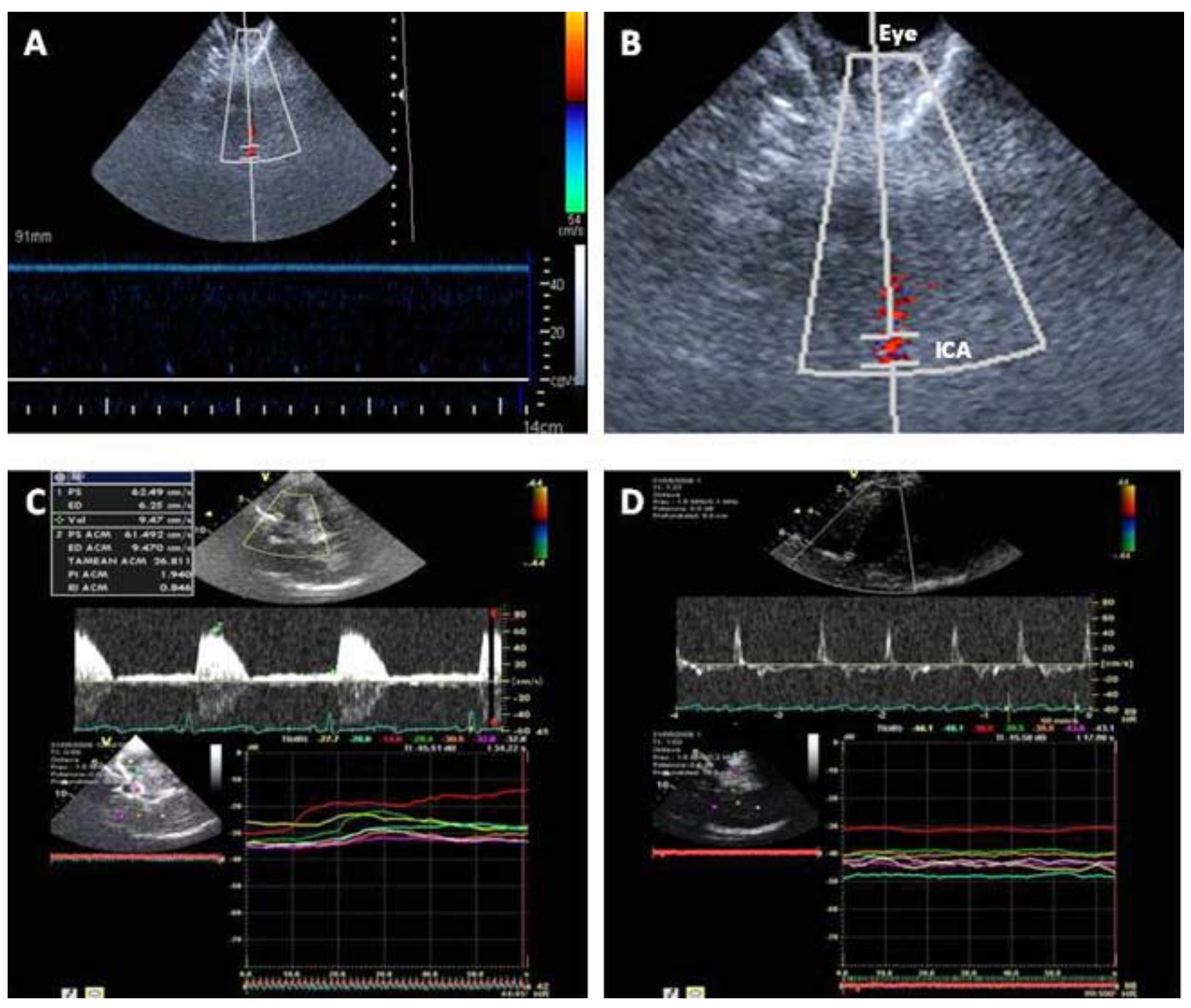

Fig. (1). Transcranial duplex sonography: A: transorbital approach with systolic spikes in the Doppler signal. B: transorbital plane with eye ball and colour coded internal carotid artery (ICA) segment magnified from A. C: middle cerebral artery (MCA) sonographic pattern with high resistance flow (pulsatility index 1.9) and cerebral perfusion evaluation at predefined ROI's showing a delayed increased peak signal and time to peak intensity. D: $24 \mathrm{~h}$ later, MCA sonographic pattern with reversed diastolic flow and cerebral perfusion evaluated at bilateral thalamus, bilateral lentiform nucleus and contralateral white matter shows no bolus-like curve progression.

finding is the absence of any intracranial filling of the internal carotid and vertebral arteries [30], (see Fig. 3). However, the procedure is cumbersome, invasive and potentially harmful to the residual organ function, and consequently its diagnostic use for the determination of brain death is not approved in many countries. The loss of intracranial perfusion could also be investigated by means of magnetic resonance imaging (MRI), MR angiography (MRA), computed tomography (CT) and CT angiography (CTA) [31]. In France, for example, the diagnosis of brain death is accepted with a CTA related 7-point score [31]. However, though the above methods are interesting and promising since they are widely available and less invasive, the techniques could not be recommended due to a lack of scientific validation to date [9].
Single photon emission computer tomography (SPECT) with radioactively labelled tracers, such as ${ }^{99 \mathrm{~m}}$ Technetium labelled HMPAO or ECD, is a further cerebral perfusion imaging technique applied in brain death assessment [32, 33]. In case of brain death, any signal from the intracranial compartment is lacking with normal uptake in the tissue outside the brain (Fig. 4).

\section{Electrophysiological Tests for Brain Death Confirmation}

Functional ancillary methods include electroencephalography (EEG) and evoked potentials (somatosensory and brainstem auditory evoked potentials; SSEP and BAEP, respectively). These methods have a considerable variation relating to inter-rater agreements as well as technical limitations in common. 

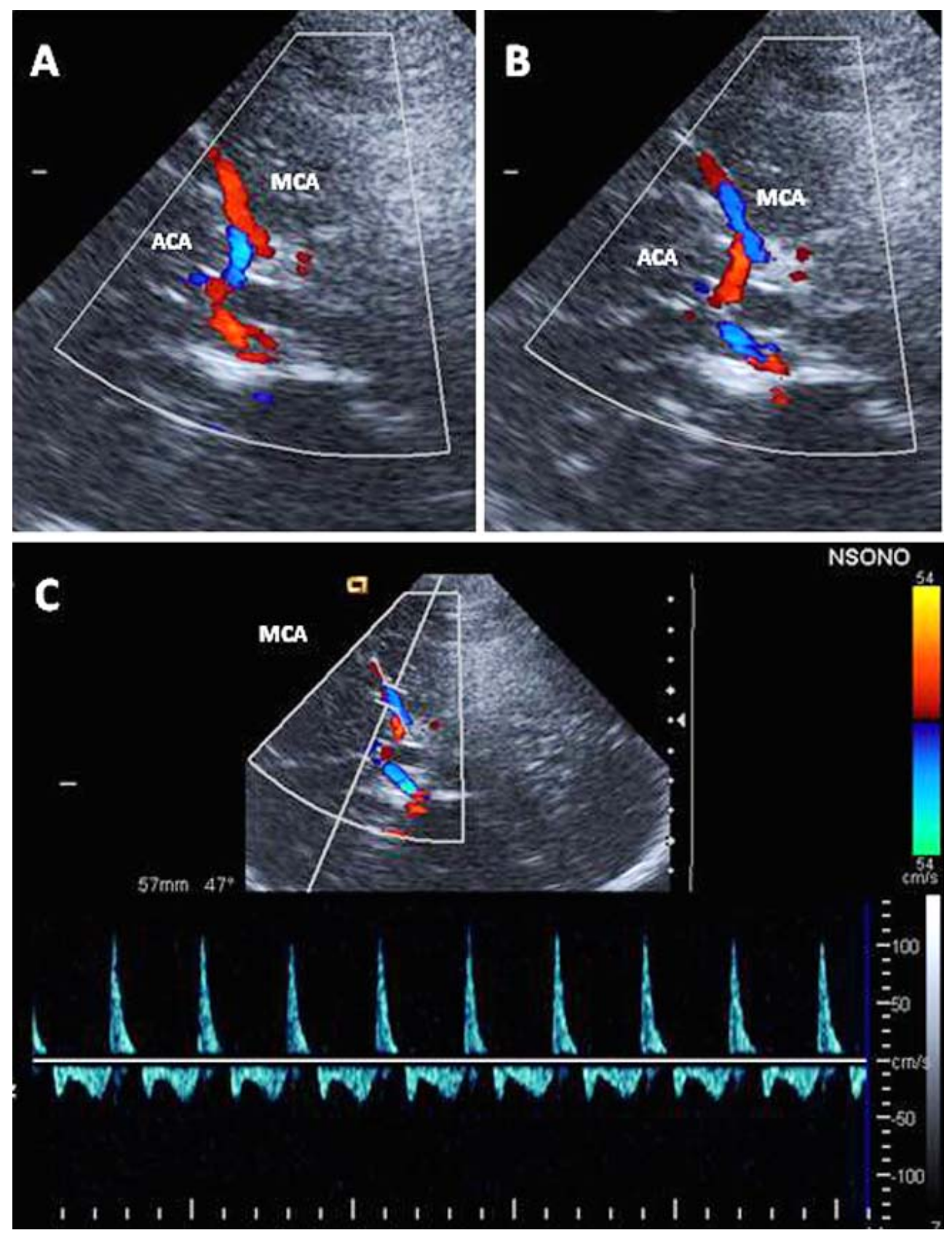

Fig. (2). Transcranial colour coded duplex sonography with oscillatory colour signal (MCA, ACA - changing from blue to red, and vice versa; A and B). C: Same patient's sonographic pattern together with CCA-Doppler signal.

Electroencephalography records cortical brain activity, however, no infratentorial or thalamic (in-) activity can be demonstrated using the method. Furthermore, in the ICU setting, the presence of a variety of electrical instruments often result in EEG artifacts, which cannot always be clearly distinguished from residual brain activity. Another limitation is the effect of sedatives and other drugs, which may induce a reversible flat or even isoelectric EEG. Screening tests for drugs may be helpful for identifying substances that mimic brain death, e.g. intoxications with barbiturates or tricyclic antidepressants. However, serum concentrations which exclude a false positive isoelectric flat line EEG are not generally accepted, because the dose-effect relationship of drugs may be heterogeneous in severe brain injury, and some toxins may not be detected on routine screening tests [1].
Thus, EEG seems to be predominantly useful in primarily infratentorial brain damage and certain clinical conditions, as, for example, following decompressive craniectomy or in neonatal patients, where other confirmatory tests maybe indecisive. When EEG is performed by an experienced investigator, the absence of electrical brain activity may be considered as a reliable tool for demonstrating irreversible loss of supratentorial/cortical brain function. Although, residual EEG activity has been found in patients, who have been diagnosed as brain dead [see 16].

SSEP (cerebral N20 and spinal N13 response with median nerve stimulation) and BAEP (wave I - V) have been recommended in several guidelines. The procedures are being employed and implemented in some countries to 

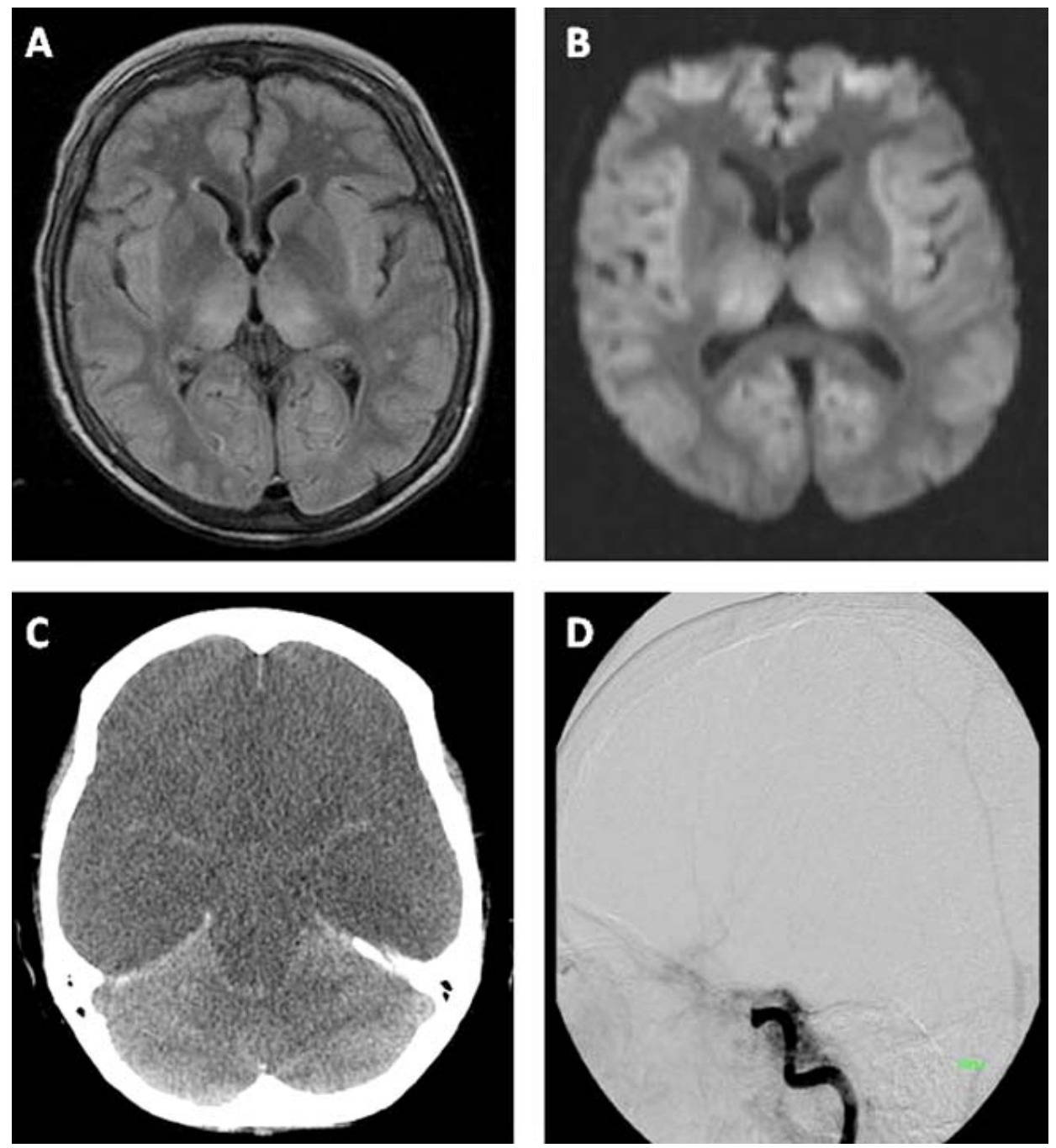

Fig. (3). A: MRI (FLAIR) and B: Diffusion weighted imaging (DWI) of a patient with metabolic encephalopathy showing hyperintense bithalamic and cortical structures. C: 24 h later same patient with progressive brain edema and signs of herniation. D: Angiography (ICA) showing intracranial stop of contrast flow determining CCA. Courtesy by the Institute of Diagnostic and Interventional Radiology at the University Hospital Jena.

confirm the irreversibility of the loss of the total brain function. Brain stem function loss in primarily supratentorial or secondary brain damage with suspected craniocaudal progress can be demonstrated using these methods. An advantage of the methods lies in the fact that both SSEP and BAEP are only slightly influenced by drugs and metabolic derangements as compared to EEG [34, 35]. On the other hand, both methods have limited suitability with regard to technical, anatomical and pathophysiological aspects. Evoked potentials do not cover more than the specific areas of interest (i.e. brainstem or sensory cortex), thus, inner ear trauma or cervical cord or peripheral nerve lesions may limit their use in the diagnosis of brain death. Conversely, the preservation of electrophysiological signals, such as N18 in SSEP [36], or other signs of electrical brain activity would definitely exclude brain death, whereas its loss would strongly suggest it.

\section{DOCUMENTATION}

The results of a clinical brain death determination must be documented, signed and dated by every performing physician. The document has to include: 1) the etiology of a severe brain injury sufficient to cause brain death and the exclusion of reversible causes, 2) the clinical findings of deep coma and absence of brain stem reflexes including apnea, 3) the confirmation of the irreversibility of these findings by repetitive examinations after a time interval or by ancillary tests or both. The time point, when confirmatory testing is concluded, constitutes the time of the patient's documented death.

\section{CONCLUSION}

The diagnosis of brain death both in adults and children primarily consists of a careful anamnesis to clarify etiology and to exclude reversible causes of coma, a neurological examination and a confirmation of the irreversibility of the brain functional loss. Confirmatory tests are performed in order to verify brain death alternatively or complementary to an observation time and in special conditions, when clinical criteria cannot be applied reliably. In case of any doubt, repeat (clinical) examinations of brain death are absolutely mandatory in order to avoid false positive results. 
Table 1. Pitfalls and Open Questions in Brain Death Diagnosis (Adopted from [5])

\begin{tabular}{|c|c|c|}
\hline Prerequisites & Clinical Diagnosis & Irreversibility \\
\hline \hline Normal/no brain imaging & "Lazarus Sign", other spinal motor signs & Duration of observational period after $1^{\text {st }}$ protocol \\
\hline $\begin{array}{c}\text { Effect of neuromuscular blockade, sedative } \\
\text { or analgesic agents }- \text { role of antidots }\end{array}$ & Profuse sweating, blushing, tachycardia & $\begin{array}{c}\text { Doppler with residual flow (with/without } \\
\text { craniotomy or ventricular drainage) }\end{array}$ \\
\hline $\begin{array}{c}\text { Mild hypothermia }\left(>32^{\circ} \mathrm{C} ;<34^{\circ} \mathrm{C}\right) \\
\text { Secondary hypothermia }\end{array}$ & Preexisting pupilary abnormalities (post-surgery) & $\begin{array}{c}\text { Residual EEG-activity } \\
\text { ICU-related EEG-artifacts }\end{array}$ \\
\hline $\begin{array}{c}\text { Metabolic/endocrine derangements } \\
- \text { cut-off values? }\end{array}$ & $\begin{array}{c}\text { Lid edema/chemosis } \\
\text { Eevere facial trauma, skull base fractures } \\
\text { metabolic derangements }\end{array}$ \\
\hline $\begin{array}{c}\text { Toxicological screening in suspected intoxication } \\
\text { Respiratory like-movements (Shoulder } \\
\text { elevation/adduction, back arching) during } \\
\text { apnea testing }\end{array}$ & $\begin{array}{c}\text { Angiography: how much time } \\
\text { to elapse before concluding CCA? }\end{array}$ \\
\hline
\end{tabular}
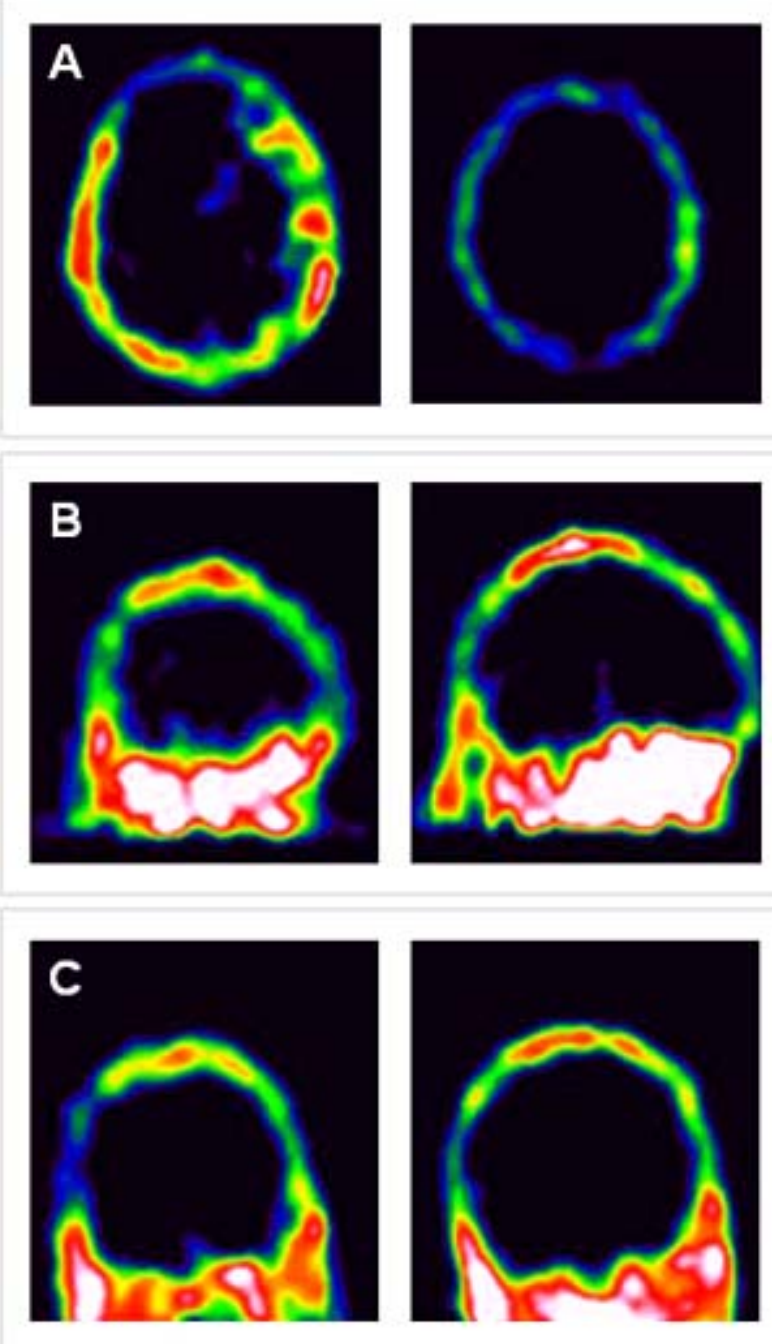

Fig. (4). Examples of a HMPAO ${ }^{99 \mathrm{~m}}$ Technetium SPECT imaging of a brain death patient showing a complete lack of brain perfusion with remaining signal of extracerebral tissue. A: Transversal plane. B: Sagittal plane. C: Coronal plane. Courtesy by the Institute of Nuclear Medicine, Vivantes Berlin, Dr. A. Götsche.

Despite numerous improvements relating to techniques such as Doppler sonography or EEG, the diagnosis of the brain death in the clinical situation remains complex in terms of clinical signs and ancillary tests (see also Table 1).

There is a need to further establish and validate less invasive, and thus, maybe more suitable methods, not only to determine brain perfusion arrest by using ultrasound perfusion imaging, or MRA and CTA, but also to investigate specificity and sensitivity of a combined use of electrophysiological methods (SSEP/BAER/EEG) and CCA studies (ultrasound perfusion imaging and MRA/CTA).

Furthermore, taking new developments into account, it seems desirable to then re-evaluate the definition of brain death in terms of clinical examination and confirmatory testing, and to specify further national and/or international guidelines in the near future. Finally, performed by designated and experienced physicians with a high proficiency in determination of brain death, uniformly implemented guidelines will provide a reliable basis to deal with this important issue in the clinical routine.

With regard to different guidelines worldwide and to new technical approaches in this area the development of an international standard in the evaluation of brain death should strongly be considered.

\section{CONFLICT OF INTEREST}

There are no conflicts of interest of the authors.

\section{ACKNOWLEDGEMENT}

We thank Nasim Kroegel for careful revision of the manuscript.

\section{REFERENCES}

[1] Wijdicks EF. The diagnosis of brain death. N Engl J Med 2001; 344: $1215-21$.

[2] A definition of irreversible coma. Report of the ad hoc committee of the harvard medical school to examine the definition of brain death. JAMA 1968; 205: 337-40.

[3] Wijdicks EF. The neurologist and harvard criteria for brain death. Neurology 2003; 61: 970-76.

[4] Wijdicks EF. Brain death worldwide: Accepted fact but no global consensus in diagnostic criteria. Neurology 2002; 58: 20-25.

[5] Wijdicks EF. Determining brain death in adults. Neurology 1995; 45: 1003-11.

[6] Bershad EM, Humphreis WE, 3rd, Suarez JI. Intracranial hypertension. Semin Neurol 2008; 28: 690-702.

[7] The quality standards subcommittee of the american academy of neurology. Practice parameters for determining brain death in adults (summary statement). Neurology 1995; 45: 1012-14. 
[8] Haupt WF, Rudolf J. European brain death codes: A comparison of national guidelines. J Neurol 1999; 246: 432-37.

[9] Greer DM, Varelas PN, Haque S, Wijdicks EF. Variability of brain death determination guidelines in leading US neurologic institutions. Neurology 2008; 70: 284-89.

[10] Wijdicks EF, Rabinstein AA, Manno EM, Atkinson JD. Pronouncing brain death: Contemporary practice and safety of the apnea test. Neurology 2008; 71: 1240-44.

[11] Varelas PN, Abdelhak T, Hacein-Bey L. Withdrawal of lifesustaining therapies and brain death in the intensive care unit. Semin Neurol 2008; 28: 726-35.

[12] Wijdicks EF. In search of a safe apnea test in brain death: Is the procedure really more dangerous than we think? Arch Neurol 1995; 52: 338-39.

[13] Saposnik G, Bueri JA, Maurino J, et al. Spontaneous and reflex movements in brain death. Neurology 2000; 54: 221-23.

[14] Bundesärztekammer WBd: Richtlinien zur Feststellung des Hirntodes. Dt Ärztebl 1998; 95: A1861-68.

[15] Haupt WF, Hofling W. The diagnosis of brain death: Medical and legal aspects with special reference to the german transplantation law (tpg). Fortschr Neurol Psychiatr 2002; 70: 583-90.

[16] Young GB, Shemie SD, Doig CJ, Teitelbaum J. Brief review: the role of ancillary tests in the neurological determination of death. Can J Anesth 2006; 53: 533-39.

[17] Ducrocq X, Hassler W, Moritake K, et al. Consensus opinion on diagnosis of cerebral circulatory arrest using Doppler-sonography. Task Force Group on cerebral death of the Neurosonolgy Research Group of the World Federation of Neurology. J Neurol Sci 1998; 159: $145-50$

[18] Petty GW, Mohr JP, Pedley TA et al. The role of transcranial Doppler in confirming brain death: sensitivity, specificity, and suggestions for performance and interpretation. Neurology. 1990; 40: $300-3$.

[19] Lampl Y, Gilad R, Eschel Y, et al. Diagnosing brain death using the transcranial Doppler with a transorbital approach. Arch Neurol 2002; 59: 58-60.

[20] Domínguez Roldan JM, Jiménez González PI, García Alfaro C, et al. Diagnosis of brain death by transcranial Doppler sonography: solutions for cases of difficult sonic windows. Transplant Proc 2004; 36: 2896-7.

[21] Conti A, Iacopino DG, Spada A, et al. Transcranial doppler ultrasonography in the assessment of cerebral circulation arrest: improving sensitivity by transcervical and transorbital carotid insonation and serial examinations. Neurocrit Care 2009; 10: 32635.

[22] Llompart-Pou JA, Abadal JM, Velasco J, et al. Contrast-enhanced transcranial color sonography in the diagnosis of cerebral circulatory arrest. Transplant Proc 2009; 41: 1466-8.
[23] Abadal JM, Llompart-Pou JA, Homar J, et al. Ultrasonographic cerebral perfusion in assessment of brain death: a preliminary study. J Ultrasound Med 2008; 27: 791-4.

[24] Della Martina A, Meyer-Whiete K, Allemann E, et al. Ultrasound contrast agents for brain perfusion imaging and ischemic stroke therapy. J Neuroimaging 2005; 15: 217-32.

[25] Seidel G, Meairs S. Ultrasound contrast agents in ischemic stroke. Cerebrovasc Dis 2009; 27 Suppl 2: 25-39.

[26] Bartels E, Bittermann HJ. Transcranial contrast imaging of cerebral perfusion in patients with space-occupying intracranial lesions. J Ultrasound Med 2006; 25: 499-507.

[27] Eyding J, Krogias C, Schollhammer M, et al. Contrast-enhanced ultrasonic parametric perfusion imaging detects dysfunctional tissue at risk in acute MCA stroke. J Cereb Blood Flow Metab 2006; 26: 576-82.

[28] Meyer-Whiete K, Cangür H, Schindler A, et al. Ultrasound perfusion imaging: Determination of thresholds for the identification of critically disturbed perfusion in acute ischemic stroke - a pilot study. Ultrasound Med Biol 2007; 33: 851-56.

[29] Krogias C, Postert T, Meves S, et al. Semiquantitative analysis of ultrasonic cerebral perfusion imaging. Ultrasound Med Biol 2005; 31: $1007-12$

[30] Braun M, Ducrocq X, Huot JC, et al. Intravenous angiography in brain death: report of 140 patients. Neuroradiology 1997; 39: 4005.

[31] Frampas E, Videcoq M, de Kerviler E, et al. CT Angiography for brain death diagnosis. Am J Neuroradiol 2009; 30: 1566-70.

[32] Spieth ME, Ansari AN, Kawada TK, Kimura RI, Siegel ME. Direct comparison of Tc-99m DPTA and Tc-99m HMPAO for evaluating brain death. Clin Nucl Med 1994; 19: 867-72.

[33] Bertagna F, Barozzi O, Puta E et al. Residual brain viability, evaluated by $99 \mathrm{~m}$ Tc-ECD SPECT in patients with suspected brain death and with confounding clinical factors. Nucl Med Comm 2009; 30: 815-21.

[34] Pratt H, Aminoff M, Nuwer MR, Starr A. Short-latency auditory evoked potentials. The international federation of clinical neurophysiology. Electroencephalogr Clin Neurophysiol Suppl 1999; 52: 69-77.

[35] Mauguiere F, Allison T, Babiloni C, et al. Somatosensory evoked potentials. The international federation of clinical neurophysiology. Electroencephalogr Clin Neurophysiol Suppl 1999; 52: 79-90.

[36] Sonoo M, Tsai-Shozawa Y, Aoki M et al. N18 in median somatosensory evoked potentials: a new indicator of medullary function useful for the diagnosis of brain death. JNNP 1999; 67: 374-78. 